\title{
Exploring Adaptive Data Centers through Cooperative Symbiotic Networking
}

\author{
Paskorn Champrasert and Junichi Suzuki \\ Department of Computer Science \\ University of Massachusetts, Boston \\ \{paskorn,jxs\}@cs.umb.edu
}

\begin{abstract}
This paper describes a biologically-inspired architecture, called SymbioticSphere, which allows large-scale data centers to autonomously adapt to dynamic environmental changes. SymbioticSphere follows biological principles such as decentralization, natural selection and symbiosis to design data centers (application services and middleware platforms). Each application service and middleware platform is designed as a biological entity and implements biological behaviors such as migration, replication death and energy exchange. Services and platforms cooperatively invoke their behaviors to improve their adaptability. This paper presents a set of behaviors for services and platforms, and describes how services and platforms act and interact with each other. Simulation results show that services and platforms autonomously adapt to dynamic network conditions (e.g., user location, network traffic, and resource availability) by invoking their behaviors suitable for the conditions.
\end{abstract}

\section{Introduction}

Data centers have become integral components to operate large-scale network applications. Since they are rapidly increasing in complexity and scale, they face several challenges, particularly autonomy and adaptability. Data centers are expected to autonomously adapt to dynamic conditions in the network (e.g., network traffic and resource availability) in order to improve user experience, expand operational longevity and reduce maintenance cost $[1,2]$.

In order to meet these challenges in data centers (i.e., autonomy and adaptability), the authors of the paper propose to apply key biological principles and mechanisms to design data centers. This is motivated by the observation that various biological systems have already developed the mechanisms to achieve autonomy and adaptability. For example, bees act autonomously, influenced by local environmental conditions and local interactions with other bees. A bee colony adapts to dynamic environmental conditions. When the amount of honey in a hive is low, many bees leave the hive to gather nectar from nearby flowers. When the hive is full of honey, most bees remain in the hive and rest.

SymbioticSphere is an architecture that applies biological principles and mechanisms to design data centers. In SymbioticSphere, each data center consists of two major components: application services and middleware platforms. SymbioticSphere models the two components as different biological species. Individual application services and middleware platforms are modeled as biological entities, analogous to bees in a bee colony. They are designed to follow several biological principles such as decentralization, natural selection and symbiosis. An application service is designed as a software agent. Each agent implements a functional service (e.g., web service) and biological behaviors such as energy exchange, replication, death and migration. A middleware platform runs on a network host and operates agents. Each platform provides runtime services that agents use to perform their services and behaviors, and implements biological behaviors such as replication, death and energy exchange.

Agents and platforms periodically sense their surrounding environmental conditions such as network traffic and resource availability, and adaptively perform their behaviors suitable for the conditions. For example, agents may invoke the migration behavior for moving towards network hosts that accept a large number of user requests for their services. This leads to the adaptation of agent locations, and agents can reduce response time for users. Platforms may invoke the replication behavior for placing additional platforms on neighboring network hosts. This leads to the adaptation of platform availability, and platforms can make more resources available for agents.

In addition to these (regular) behaviors, each agent/platform implements a special type of behaviors, called symbiotic behaviors. Each symbiotic behavior is a sequence of regular behaviors that agents and platforms perform in order. Agents and platforms are designed to adapt to dynamic network environments by performing regular behaviors, as described above; however, regular behaviors of one species (e.g., agents) can degrade the adaptation of other species (e.g., platforms) in some circumstances. For example, if too many agents migrate toward a user, the platforms running close from the user have a risk to crash due to overloading or resource extinction. Symbiotic behaviors are intended to augment the adaptability of agents and platforms by allowing the two species to cooperate for pursuing their mutual benefits.

This paper describes the regular and symbiotic behaviors in SymbioticSphere and evaluates their impacts on the adaptability of data centers (i.e., agents and platforms). Simulation results show that agents and plat- 
forms autonomously adapt to dynamic environmental conditions (e.g., user location, network traffic and resource availability) by using their regular behaviors. Simulation results also show that symbiotic behaviors improve the adaptability of agents and platforms.

\section{Design Principles in SymbioticSphere}

In SymbioticSphere, agents and platforms are designed based on the following principles.

(1) Decentralization: There are no central entities to control and coordinate agents/platforms (i.e., no directories and no resource managers). Decentralization allows agents/platforms to be scalable and survivable by avoiding a single point of performance bottlenecks and failures.

(2) Autonomy: Agents and platforms sense their local network environments, and based on the sensed environmental conditions, they autonomously behave, and interact with each other without any intervention from/to other agents, platforms and human users/administrators.

(3) Natural selection: Agents and platforms store and expend energy for living. Each agent gains energy in exchange for performing its service to other agents or human users, and expends energy to use network and computing resources. Each platform gains energy in exchange for providing resources to agents, and periodically evaporates energy. The abundance or scarcity of stored energy triggers natural selection of agents/platforms. For example, an abundance of stored energy indicates higher demand for an agent/platform; thus the agent/platform replicates itself to increase its availability. A scarcity of stored energy (an indication of lack of demand) causes death of the agent/platform. Like in biological natural selection where more favorable species in an environment becomes more abundant, the population of agents/platforms dynamically changes based on the demands for them.

(4) Emergence: Agents and platforms behave against dynamic environmental conditions (e.g. user demands and resource availability). For example, an agent may invoke migration behavior to move towards a platform that forwards a large number of request messages for its services. Also, a platform may replicate itself on a neighboring host whose resource availability is high. Through collective behaviors and interactions of individual agents and platforms, desirable system characteristics such as adaptability and survivability emerge in a swarm of agents and platforms. Please note that the desirable characteristics are not present in any single agent/platform.

(5) Symbiosis: Although competition for food and terrain always happens in the biological world, several entities establish relationships to avoid excessive competitions and support with each other to survive. In SymbioticSphere, agents and platforms are modeled as different biological species. Agents and platforms cooperate with each other for pursuing their mutual benefits (e.g., gaining more energy to survive) and improve their adaptability.

\section{SymbioticSphere}

\subsection{The Architecture of SymbioticSphere}

Figure 1 shows the architecture of SymbioticSphere. Agents and platforms are modeled as different biological species. As a living entity, the ultimate goal of each species is to survive for a long time by balancing its energy gain and population.

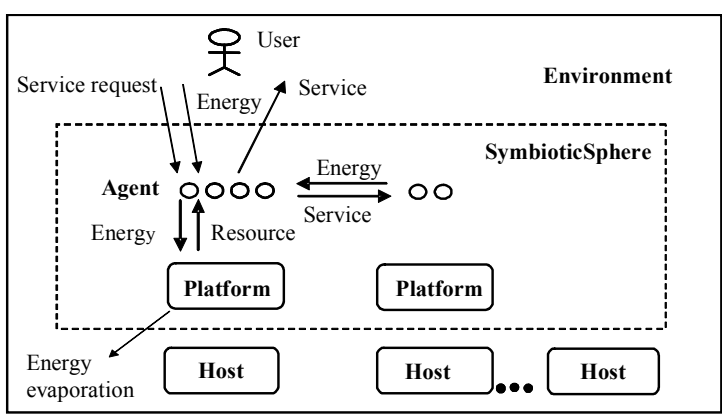

Figure 1. Energy Exchange in SymbioticSphere

SymbioticSphere follows ecological principles to design energy exchange among agents, platforms and environment. It models a user as the Sun, agents as producers, and platforms as consumers ${ }^{1}$. Similar to the Sun, users have unlimited amount of energy. Each agent gains energy from users ${ }^{2}$ and transfers $10 \%$ of its energy level to an underlying platform for consuming resources provided by the platform. Each platform gains energy from agents and evaporates $10 \%$ of its energy level to the environment. This energy exchange rule follows an ecological fact that about $10 \%$ of the energy maintained by producer species goes to consumer species [3]. Due to space limitation, see [4] for more details on energy exchange in SymbioticSphere.

\subsection{Agents}

Each agent consists of three parts: attributes, body and behaviors. Attributes carry descriptive information regarding the agent, such as agent ID, energy level and

\footnotetext{
${ }^{1}$ In the ecological system, producers (e.g., shrubs) convert the Sun light energy to chemical energy. The chemical energy is transferred to consumers (e.g., hares) as consumers consume producers [3].

${ }^{2}$ Each agent specifies the price (in energy units) of its service.
} 
description of a service it provides. Body implements a service that the agent provides. For example, an agent may implement a web service, while another agent may implement a physical model for scientific simulations. Behaviors implement actions that are inherent to all agents. Although SymbioticSphere defines nine standard agent behaviors [5], this paper focuses on three of them.

- Replication: Agents may make a copy of themselves as a result of abundance of energy. A replicated (child) agent is placed on the platform that its parent agent resides on, and it receives the half amount of the parent's energy level.

- Death: Agents die due to energy starvation. When an agent dies, an underlying platform removes the agent and releases all resources allocated to the agent.

- Migration: Agents may move from one platform to another.

\subsection{Platforms}

Each platform runs on a network host and operates agents. It consists of attributes, behaviors and runtime services. Attributes carry descriptive information regarding the platform, such as platform ID, energy level and health level. Health level indicates how healthy an underlying host is. It is defined as a function of three properties: resource availability on, age of and freshness of a host. Resource availability indicates how much resources are available for agents and platforms on a host. Age indicates how long a host has been alive (i.e., how much stable a host is). Freshness indicates how recently a host joined the network. After a new host joined the network, its freshness gradually decreases from the maximum value. When an unstable host resumes from a failure, its freshness starts with the value that the host has when it went down. Using age and freshness, unstable hosts and new hosts can be distinguished (Table 1). Health level affects behaviors of each platform and agent. For example, higher health level indicates higher stability of and/or higher resource availability on a host that a platform resides on. Thus, the platform may replicate itself on a healthier neighboring host.

Table 1.Freshness and Age in Different Types of Hosts

\begin{tabular}{|l||c|c|}
\hline \multicolumn{1}{|c||}{ Host Type } & Freshness & Age \\
\hline \hline Unstable host & Lower & Lower \\
\hline New host & Higher & Lower \\
\hline Stable host & Lower & Higher \\
\hline
\end{tabular}

Behaviors are the actions inherent to all platforms.

- Replication. Platforms may make a copy of themselves as a result of abundance of energy (i.e., higher demand for resources available on the platforms).
The child platform inherits the half of the parent's energy level.

- Death. Platforms die due to lack of energy. A dying platform uninstalls itself and releases all resources the platform uses. Despite the death of a platform, an underlying host remains active so that another platform can run on it in the future.

Runtime services are middleware services that agents and platforms use to perform their behaviors.

\subsection{Behavior Policies of Agents and Platforms}

Each agent/platform has policies for its behaviors. A behavior policy defines when to and how to invoke a particular behavior. Each behavior policy consists of factors $\left(\mathrm{F}_{\mathrm{i}}\right)$, which evaluate environment conditions (e.g. network traffic) or agent/platform/host status (e.g. energy level and health level). Each factor is given a weight $\left(\mathrm{W}_{\mathrm{i}}\right)$ relative to its importance. Behaviors are invoked if the weighted sum of factor values $\left(\Sigma \mathrm{F}_{\mathrm{i}} * \mathrm{~W}_{\mathrm{i}}\right)$ exceeds a threshold. The factors in agent migration behavior policy include:

- Energy Level: Agent energy level, which encourages agents to move in response to higher energy level.

- Health Level Ratio: The ratio of health level on a remote host to the local host, which encourages agents to move to platforms running on healthier hosts. This ratio is calculated with three health level properties (i.e., resource availability, freshness or age) as follows:

Health Level Ratio $=$

$\sum_{i}^{3}\left(\frac{\text { HealthLevel } \operatorname{Pr} \text { operty }_{i} \text { on remote host }- \text { HealthLevel } \operatorname{Pr} \text { operty }_{i} \text { on local host }}{\text { HealthLevel } \operatorname{Pr} \text { operty }_{i} \text { on local host }}\right)$ (1)

- Service Request Ratio: The ratio of \# of incoming service requests on a remote platform to the local platform, which encourages agents to move towards users.

- Migration Interval: Time interval to perform migration, which discourages agents to migrate too often.

If there are multiple neighboring platforms that an agent can migrate to, the agent calculates a weighted sum of the above factors for each of the platforms, and moves to a platform that generates the highest weighted sum.

The factors in agent replication behavior policy include:

- Energy Level: Agent energy level, which encourages agents to replicate themselves in response to higher energy level.

- Request Queue Length: The length of service request queue, which the local platform maintains to 
queue incoming service requests. This factor encourages agents to replicate themselves in response to higher demands.

The factors in agent death behavior policy include:

- Energy Level: Agent energy level. Agents die when they run out of their energy.

- Energy Loss Rate: The rate of energy loss in between the current and previous simulation cycles. This factor is calculated with the following equation, where $E_{t}$ and $E_{t-1}$ are the energy levels in the current and previous simulation cycles. Agents have higher risk to die in response to sharp drop in demands for their services.

$$
\text { Energy Loss Rate }=\frac{E_{t-1}-E_{t}}{E_{t-1}} \cdots \cdots(2)
$$

The factors in platform replication behavior include:

- Energy Level: Platform energy level, which encourages platforms to replicate themselves in response to higher energy level.

- Health Level Ratio: The ratio of health level on a remote host to the local host, which encourages platforms to replicate themselves on healthier neighboring hosts. This ratio is calculated with Equation (1).

- The Number of Agents: The number of agents working on each platform. This factor encourages platforms to replicate themselves in response to higher agent population on them.

If there are multiple neighboring hosts that a platform can replicate itself on, the platform places a child platform on a host whose health ratio is highest among others.

The factors in platform death behavior include:

- The Number of Agents: The number of agents running on each platform. This factor discourages platforms to die when agents run on them.

- Energy Loss Rate: The rate of energy loss in platforms. This factor is calculated with Equation 2. Platforms have higher risk to die in response to sharp drop in demands for their resources.

Each agent/platform expends energy to invoke behaviors (i.e., behavior cost) except death behavior. When the energy level of an agent/platform goes over the cost of a behavior, the agent/platform decides whether it performs the behavior by calculating a weighted sum of factors.

\subsection{Symbiotic Behaviors}

SymbioticSphere currently provides six symbiotic behaviors. Each symbiotic behavior is defined as a sequence of regular behaviors that agents and platforms perform in order. There are two types of symbiotic behaviors: agent-initiated symbiotic behaviors (A1, A2 and $\mathrm{A} 3$ behaviors) and platform-initiated symbiotic behaviors (P1, P2 and $\mathrm{P} 3$ behaviors).

A1: When an agent wants to move toward a user but there is no platform running on a neighboring host closer to the user, the agent can propose the local platform to replicate itself on the neighboring host (Figure 2 ). If the local platform's health level is low, the platform accepts the agent's proposal. The agent gives the platform the energy units of platform replication cost, and the platform replicates itself on the host that the agent wants to migrate to. As a result, the agent can migrate to the replicated (child) platform and improve response time. The platform improves its health level because resource availability becomes higher.

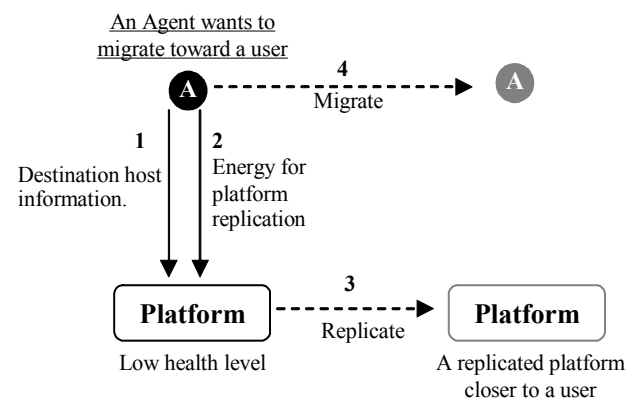

Figure 2. Symbiotic Behavior A1

A2: When an agent is dying due to energy starvation, the agent can ask the local platform to shoulder agent migration cost so that the agent can migrate to a platform on a healthier host (i.e., a platform less crowded with agents) (Figure 3). If the local platform's health level is low, the platform agrees with the agent. As a result, the agent can have a higher chance to receive more service requests (i.e., energy) from users and survive longer. The platform improves its health level because resource availability becomes higher.

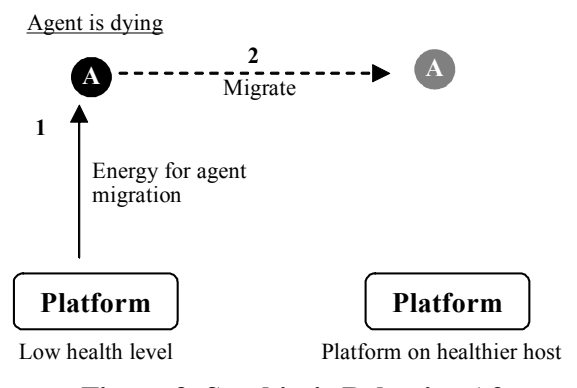

Figure 3. Symbiotic Behavior A2

A3: When an agent is dying due to energy starvation, the agent can ask the local platform to shoulder agent migration cost so that the agent can migrate to a neighboring platform closer to a user (Figure 4). If the local platform's health level is low, the platform agrees with the agent. As a result, the agent can improve re- 
sponse time. The platform improves its health level because resource availability becomes higher.

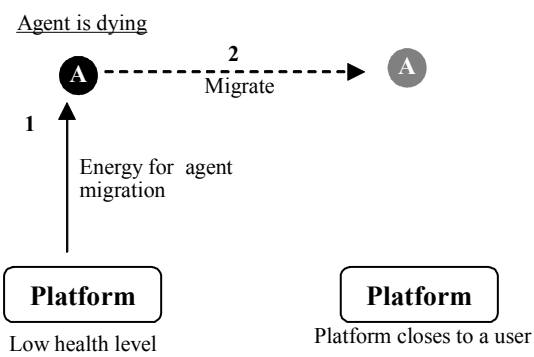

Figure 4. Symbiotic Behavior A3

P1: When a platform replicates on a healthier host, the platform can propose an agent working on it to migrate to the replicated (child) platform (Figure 5). If the agent's energy level is low, it accepts the platform's proposal. The platform gives the agent the energy units of agent migration cost, and the agent migrates. As a result, the parent platform increases its health level because resource availability becomes higher. The child platform can survive longer because it gains energy from the migrating agent. On its destination platform (i.e., platform less crowded with agents), the agent can have a higher chance to receive more service requests (i.e., energy) from users and survive longer.

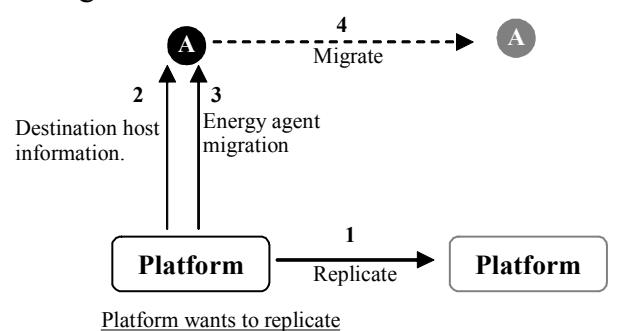

Figure 5. Symbiotic Behavior P1

P2: When a platform has very low resource availability the local host has a risk to crash due to overloading, the platform can propose a local agent to migrate to a platform on a healthier host (Figure 6.). If the agent's energy level is low, it accepts the platform's proposal. The platform gives the agent the energy units of agent migration cost, and the agent migrates. As a result, the platform increases its health level. The local agent can migrate to a platform on healthier host. As a result, the agent and platform can reduce the risk to be wiped out due to the local host crash.

P3: When a platform is dying due to energy starvation, the platform can propose the local agents to shoulder platform replication cost so that the platform can replicate itself on a host closer to a user (Figure 7). If the platform dies, the agents die off on the platform too. Thus, the agents accept the platform's proposal, and some of them migrate to a replicated (child) platform. As a result, the migrating agents gain more energy from a user (i.e., survive longer) and improve response time. The child platform can gain more energy from the agents and survive longer.

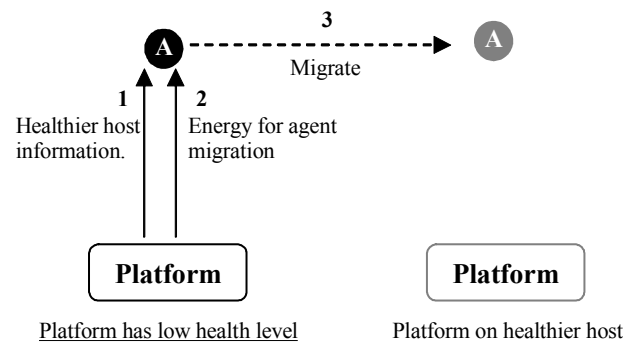

Figure 6. Symbiotic Behavior P2

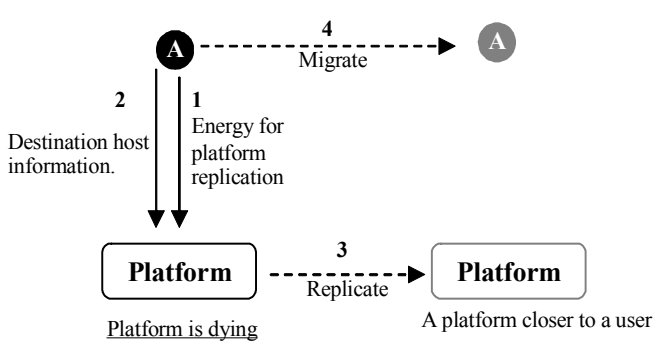

Figure 7. Symbiotic Behavior P3

\section{Simulation Results}

This section shows a set of simulation results to evaluate how agents and platforms improve their adaptability using their regular behaviors and symbiotic behaviors. Figure 8 shows a simulated network. A data center consists of hosts connected in a 7 x 7 grid topology, and users send service requests to agents via user access point. This paper assumes that a single (virtual) user runs on the access point, and it emulates multiple users to send service requests. At the beginning of a simulation, one agent and one platform are deployed on a host that is furtherest from a user.

Each host has $256 \mathrm{MB}$ memory space ${ }^{3}$. Out of the space, an operating system and Java VM consume 128 and $64 \mathrm{MB}$, respectively. The remaining space is available for a platform and agents on each host. Each agent and platform consumes 5 and $20 \mathrm{MB}$, respectively. This assumption is obtained from a prior empirical experiment [5]. Each simulation runs for 24 hours in simulation time.

Each host operates in active or inactive state. When a platform works on a host, the host is in active state and consumes $60 \mathrm{~W}$ power. The host goes to inactive state when a platform dies on it. An inactive host consumes $5 \mathrm{~W}$ power. This assumption on power consump-

\footnotetext{
${ }^{3}$ Currently, memory availability represents resource availability.
} 
tion is obtained from [6]. A host can become active from inactive state using the Wake On LAN (WOL) technology [7]. When a platform replicates itself on an inactive host, the platform sends a WOL packet to the host to wake it up.

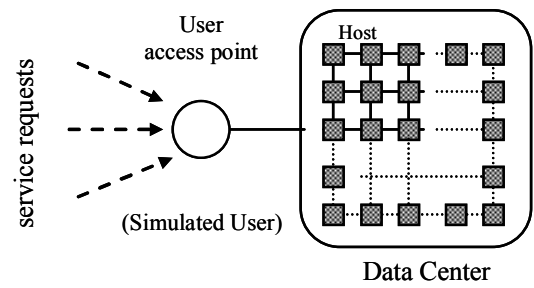

Figure 8. Simulated Network

Figure 9 shows how a user changes service request rate over time. This service request rate is taken from a workload trace of the 1998 Olympic official website [8]. The peak workload is 9,600 requests $/ \mathrm{min}$.

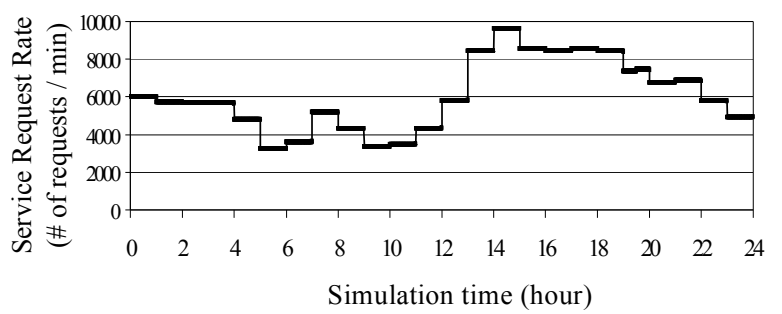

Figure 9. Service Request Rate

Figure 10 shows a pseudo code to run users, agents and platforms in each simulation cycle.

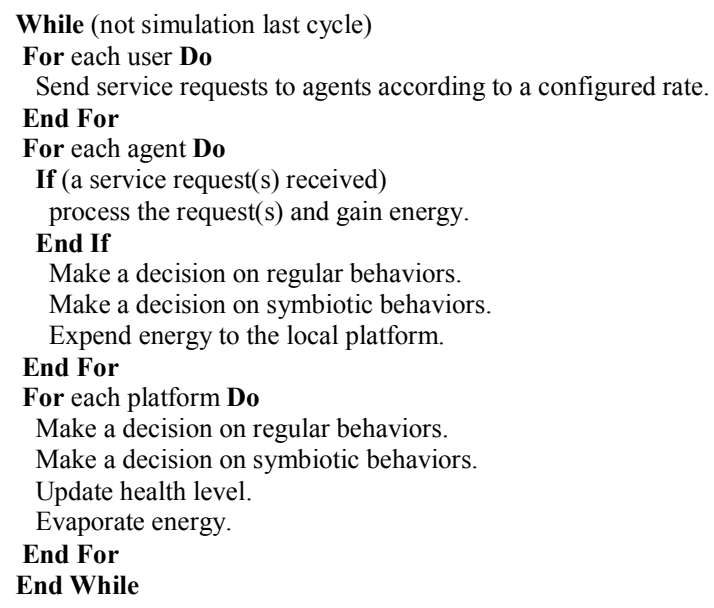

Figure 10. Pseudo Code of Simulation Cycle

In this paper, adaptability is defined as service adaptation and resource adaptation. Service adaptation is the set of activities to adaptively improve the quality and availability of services provided by agents. The quality of services is measured as response time of agents for processing service requests from users. Service availability is measured as the number of agents. Resource adaptation is the set of activities to adaptively improve resource availability and resource efficiency. Resource availability is measured as the number of platforms that makes resources available for agents. Resource efficiency indicates how many service requests can be processed per resource utilization of agents and platforms.

\subsection{Evaluation of Regular Behaviors}

This section evaluates how agents and platforms autonomously adapt to dynamic environmental conditions by using regular behaviors.

Figure 11 shows how service availability (i.e., the number of agents) and resource availability (i.e., the number of platforms) change dynamically. Starting with an agent and a platform at 0:00, they change their populations through replication in order to handle the demand placed on them $(6,000$ requests $/ \mathrm{min})$. When service request rate increases from $12: 00$ to 2:00, agents gain more energy form users and replicate themselves more often. In response to higher energy intake, they also transfer more energy to platforms. As a result, platforms also increase their population through replications. When service request rate decreases from 15:00, some of agents and platforms die because they cannot balance energy gain and expenditure due to less energy transfer from users. Figure 11 shows that biological mechanisms in SymbioticSphere contribute for agents and platforms to autonomously adapt their availability to dynamic demand changes.

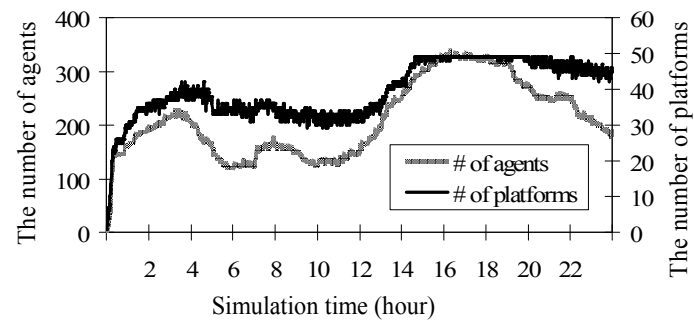

Figure 11. The Number of Agents and Platforms

Figure 12 shows the average response time and the throughput achieved by agents. In the first hour, response time is high $(25 \mathrm{sec})$ because there is only one agent and one platform needs to process 6,000 requests a minute at the beginning of a simulation. As a result, throughput does not reach $100 \%$. However, as agents and platforms replicate themselves and agents migrate towards users, the response time drops to 1 second at 2:00. (throughput reaches $100 \%$.) After 2:00, the re- 
sponse time is constantly 1 second and the throughput is constantly $100 \%$, although service request rate increases from 12:00 to 2:00. This means agents and platforms responsively change their populations and locations against demand changes. Figure 12 shows that the biological mechanisms in SymbioticSphere contribute for agents and platforms to collectively retain response time and throughput performance by adjusting their populations and locations.

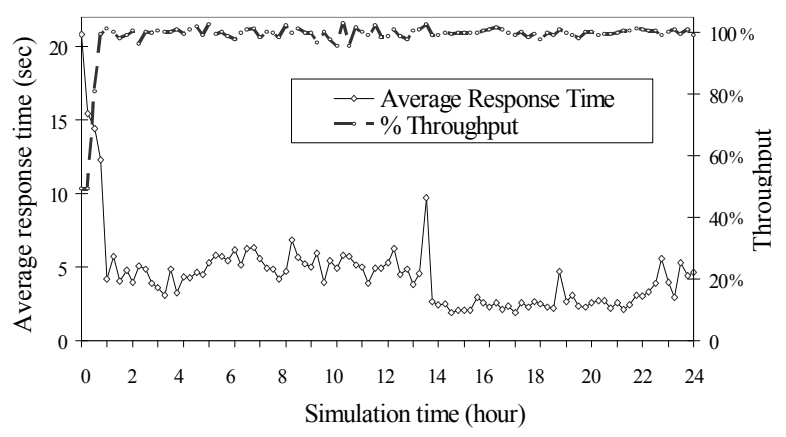

Figure 12. Response Time and Throughput

Figure 13 shows resource efficiency, which indicates how many service requests can be processed per resource unit. It is measured as (the total number of user requests processed by agents) / (the total amount resources consumed by agents and platforms). The resource efficiency of SymbioticSphere is compared with a scenario where platforms do not have biological behaviors (replication and death). This scenario simulates the always-on operation in traditional data centers. (49 platforms always run on hosts.) Since platforms of SymbioticSphere dynamically adjust resource availability according to demand changes (Figure 11), SymbioticSphere outperforms traditional data centers in resource efficiency. Figures 12 and 13 shows that the biological mechanisms in SymbioticSphere contribute for platforms to adapt resource efficiency to dynamic demand changes while helping agents improve response time and throughput performance.

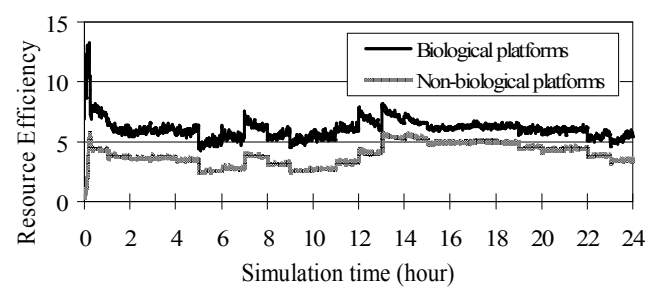

Figure 13. Resource Efficiency

\subsection{Evaluation of Symbiotic Behaviors}

This section evaluates how symbiotic behaviors contribute for agents and platforms to improve their adaptability.
Figures 15 and 16 show how symbiotic behaviors complement regular behaviors to improve the adaptability of agents and platforms. Figure 15 shows that agent-initiated symbiotic behaviors (A1, A2 and A3) contribute to improve response time performance. Figure 16 shows that platform-initiated symbiotic behaviors (P1, P2 and P3) contribute to improve the degree of load balancing. The load balancing index (LBI) indicates how workload (i.e., the number of service requests) is distributed over available platforms. (It is calculated as a standard deviation of workload; the smaller, the higher degree of load balancing.) Load Balancing Index (LBI) is measured with Equation 3 (LBI is a standard deviation of $x_{i}$ ).

$$
\text { Load Balancing Index }=\sqrt{\frac{\sum_{i}^{N}\left(X_{i}-\mu\right)^{2}}{N}}
$$

$x_{i}$ indicates (the number of messages processed by agents running on platform $i$ ) / (the amount of resources utilized by platform $i$ and agents running on platform $i$ ). $\mu$ represents the expected average of $x$, which means (the total number of messages processed by all agents) / (the total amount of resources utilized by all platforms and all agents). $N$ is the number of platforms.

However, Figures 15 and 16 do not clearly demonstrate whether symbiotic behaviors significantly improve response time and LBI results. The average response time is not significantly different when using regular behaviors only and using symbiotic behaviors as well. The LBI results contain high variances.

Therefore, this simulation study carried out an ANOVA (analysis of variance) method to evaluate how the response time and LBI results become better in the case of using symbiotic behaviors as well as regular behaviors. The ANOVA results indicate that the response time and LBI results become better with the confidence of $99.99 \%$ by using symbiotic behaviors.

Figures 17, 18, 19, 20, 21 and 22 show the average results of throughput, resource efficiency, the health level, the agent energy level, the platform energy level, and power consumption. These resulted are compared among SymbioticSphere with symbiotic behaviors (i.e. A1, A2, A3, P1, P2, and P3) and SymbioticSphere without symbiotic behaviors (i.e. normal).

Figure 17 shows the average throughput, which is the ratio of the number of processed services and the number of service requests. The result shows that agent and platforms with and without symbiotic behaviors contribute to maintain very high throughput (i.e. the average of throughput is greater than $97.8 \%$ ). 


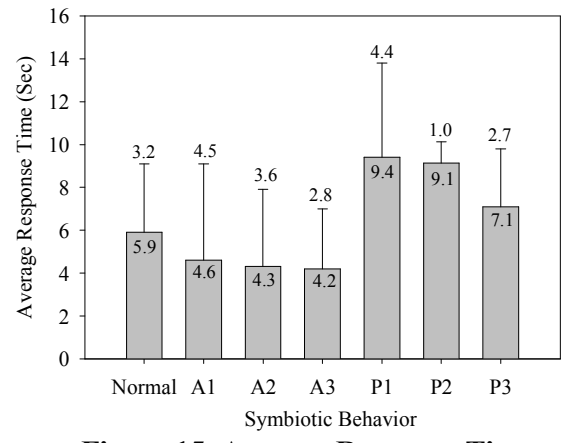

Figure 15. Average Response Time

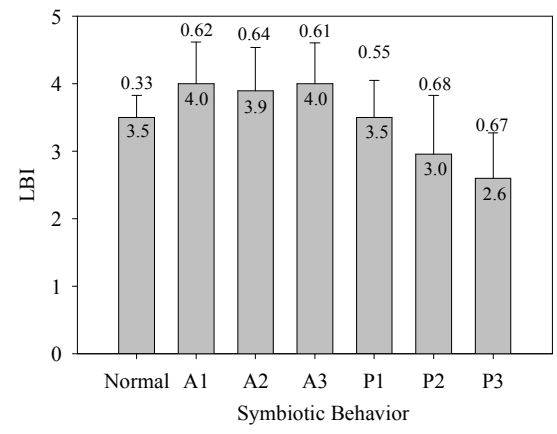

Figure 16. LBI

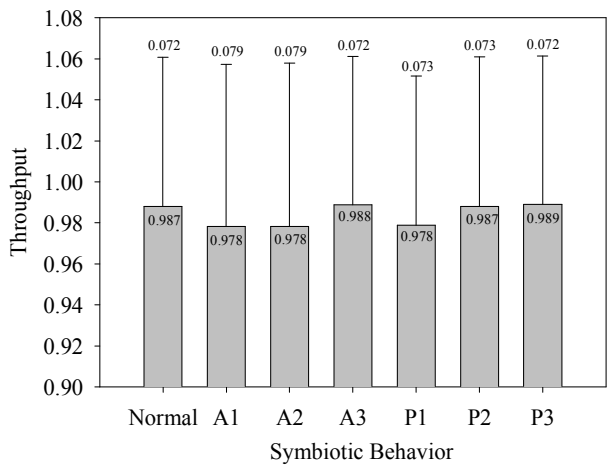

Figure 17. Throughput

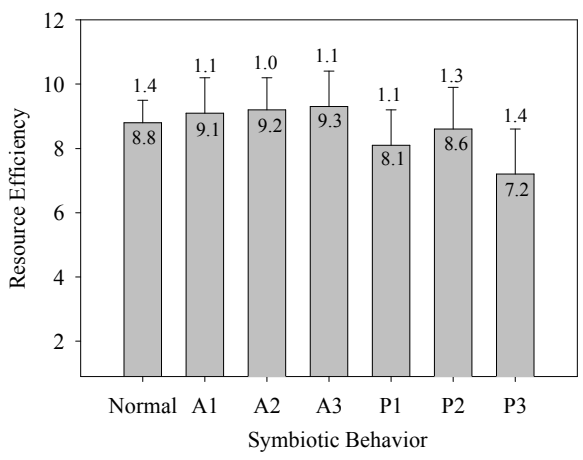

Figure 18. Resource Efficiency

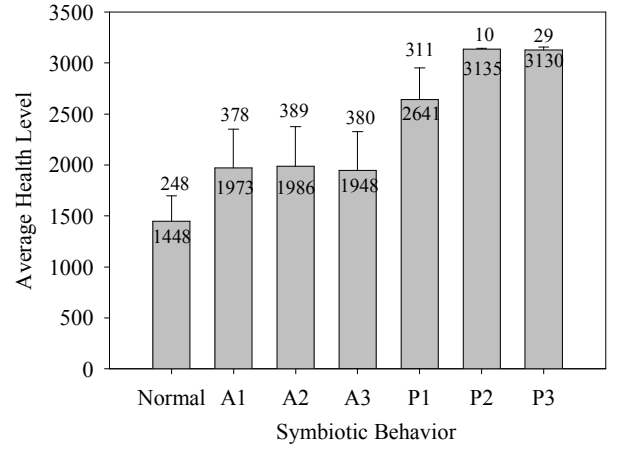

Figure 19. Average Health Level

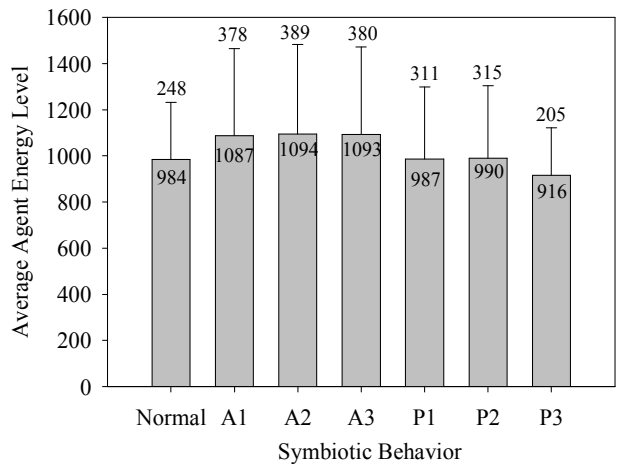

Figure 20. Average Agent Energy Level

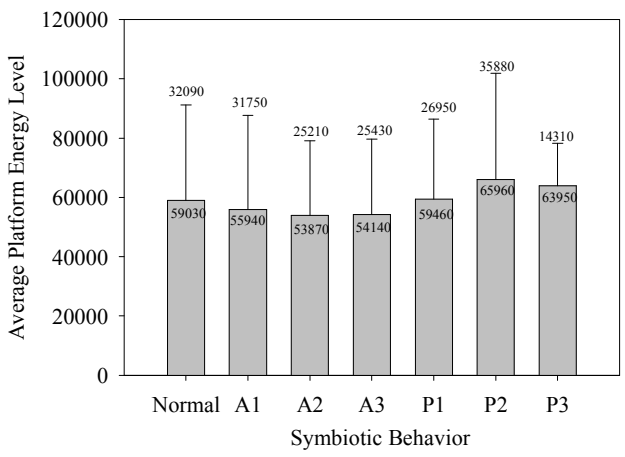

Figure 21. Average Platform Energy Level

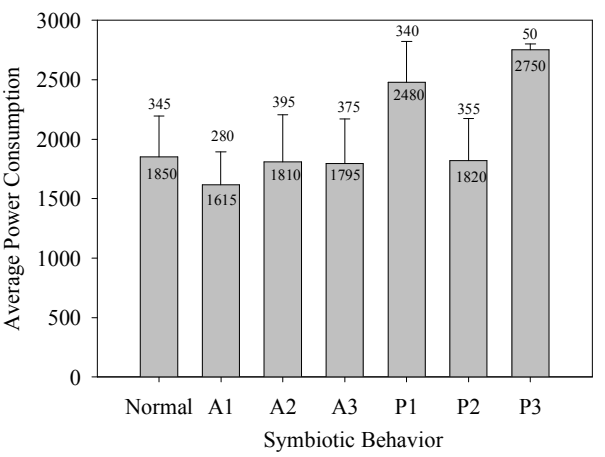

Figure 22. Average Power Consumption 
Figure 18 shows the average resource efficiency. The result shows that agents and platforms with symbiotic behavior initiated by agents (i.e. A1, A2 and A3) contribute to higher resource efficiency than the agent and platforms with regular behaviors. This happens because platforms cooperate agents to move towards a user. The number of agents on the platforms those are close to a user is very high. The platforms those are far from a user have no agent, and they die due to energy starvation. The number of platforms is reduced. The resource efficiency is improved.

Figure 19 shows the average health level. The result shows that agents and platforms with symbiotic behavior (i.e., $\mathrm{A} 1, \mathrm{~A} 2, \mathrm{~A} 3, \mathrm{P} 1, \mathrm{P} 2$ and $\mathrm{P} 3$ ) contribute to higher health level than the agent and platforms with regular behaviors. This happens because platforms cooperate agents to move to platforms working on healthier host and agents cooperate platforms to replicate to the healthier host. The average health level is increased.

Figure 20 shows the average agent energy level. The result shows that agents and platforms with symbiotic behaviors initiated by agent (i.e., A1, A2 and A3) contribute to increase agent energy level, which means agents can survive longer. This happens because platforms cooperate agents to move towards a user and gain more energy.

Figure 21 shows the average platform energy level. The result shows that agents and platforms with symbiotic behaviors initiated by platforms (i.e., P1, P2 and P3) contribute to increase platform energy level, which means platforms can survive longer. This happens because agents cooperate platforms to replicate on a host close to a user then agents can migrate to the replicated platform. Agents give more energy to platforms. The average platform energy level is increased.

Figure 22 shows the average power consumption. The result shows that that agents and platforms with symbiotic behaviors initiated by agents (i.e., A1, A2 and A3) contribute to save power consumption. This happens because the agents can move towards a user and the number of activated host (i.e. the number of platforms) is reduced. The power consumption is reduced

Figures 16-22 show that the symbiotic behaviors in SymbioticSphere contribute for agents and platforms to improve their degree of adaptability to dynamic demand changes.

Figure 23 shows the number of failed hosts. Assume that the host that has available memory space less than $5 \mathrm{MB}$ more than 15 minuets has $40 \%$ chances to be crashed. After 5 minutes failed hosts will reboot and reconnect to the data center. The result shows that that agents and platforms with symbiotic behaviors initiated by platform (i.e., P1, P2 and PA3) contribute to re- duced the number failed hosts. This happens because the agents cooperate platforms to replicate on the healthier host (P1) or agents can migrate to healthier platforms to increases the health level of the local platform (P2). The number of failed hosts is reduced.

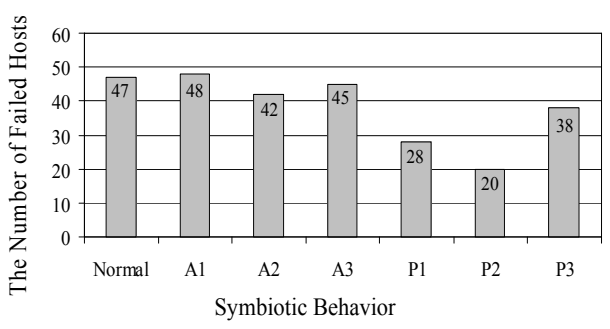

Figure 23. The Number of Failed Hosts

Figure 24 shows how the combinations of symbiotic behaviors impact the performance of agents and platforms. The performance of agents and platforms are measured with seven performance metrics of response time, throughput, LBI, resource efficiency, average platform health level, average agent energy level and average platform energy level:

$$
\text { Performance Ratio }=\sum_{i}^{7}\left(\frac{P S i-P R i}{P R i}\right) \text { (4) }
$$

$P S_{i}$ indicates performance metric I with symbiotic behaviors and $P R_{i}$ indicates performance metric $\mathrm{i}$ with out symbiotic behaviors.

Figure 24 shows that the combinations of symbiotic behaviors improve performance ratio results than single symbiotic behaviors. This is because the combinations of symbiotic behaviors can improve several performance metrics simultaneously. For example, P3A1 improve the average response time and the LBI because agents can reduce response time with $\mathrm{A} 1$ and platforms can distribute workload with P2. Hence, the performance ratio of $\mathrm{P} 3 \mathrm{~A} 1$ is higher than that of $\mathrm{P} 3$ and $\mathrm{A} 1$.

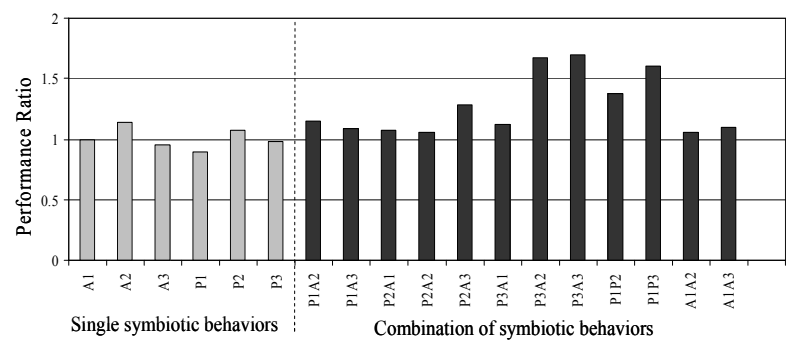

Figure 24. Average Power Consumption

\section{Related Work}


This work is an extension of previous research work $[4,9,10]$, which show agents and platforms improve their adaptability, scalability and survivability with their regular behaviors. The previous work did not investigate symbiotic behaviors. This paper shows that symbiotic behaviors complement regular behaviors to improve the adaptability of agents and platforms. This work is the first attempt to improve the adaptability of network systems (e.g., data centers) through cooperation (or symbiosis) between application components (agents) and middleware platforms.

[5] proposes biologically-inspired agents to achieve service adaptation in a decentralized manner. However, platforms are not designed to achieve resource adaptation because they are static and non-biological entities. In SymbioticSphere, both agents and platforms are biological entities, and they achieve service adaptation and resource adaptation simultaneously.

[11] and [12] implement the concept of symbiosis between different groups of peers (hosts) in peer-topeer networks. Peer groups symbiotically connect or disconnect with each other to improve the quality of query results. A special type of peers, cooperative peers, implements the symbiotic behaviors for peer group connection/disconnection. Cooperative peers do not address service adaptation and resource adaptation as SymbioticSphere does. Also, rather than a symbiosis between groups of hosts, SymbioticSphere focuses on a symbiosis between agents and platforms.

Rainbow [13] achieves both service adaptation and resource adaptation in grid computing environments. A centralized system monitor periodically inspects the current environmental conditions and performs an adaptation strategy (e.g., service migration and platform replication/death). In SymbioticSphere, agents and platform implement more adaptation strategies (agent/platform behaviors) such as agent replication and agent death. They invoke their behaviors to adapt to dynamic environmental conditions in a decentralized manner. SymbioticSphere also provides symbiotic behaviors to improve the adaptability of agents and platforms. Rainbow does not consider symbiosis (or cooperation) between services and platforms.

[14] proposes a decentralized design for adaptive data centers that guarantee response time. SymbioticSphere does not guarantee any system measures including response time because piecemeal improvement of those measures is an emergent product from collective behaviors and interactions of agents and platforms. As a result, agents and platforms can adapt to unexpected environmental changes without changing any behaviors and their policies.

\section{Conclusion}

This paper presents two different (regular and symbiotic) behaviors that agents and platforms implement in SymbioticSphere, and describes how agents and platforms act and interact with each other. Simulation results show that agents and platforms autonomously adapt to dynamic environmental conditions (e.g., user location, network traffic and resource availability) by using their regular behaviors. Simulation results also show that symbiotic behaviors improve the adaptability of agents and platforms.

\section{References}

[1] P. Dini, W. Gentzsch, M. Potts, A. Clemm, M. Yousif and A. Polze, "Internet, Grid, Self-adaptability and Beyond: Are We Ready?," In Proc. of the IEEE International Workshop on SelfAdaptable and Autonomic Computing Systems, August 2004.

[2] R. Sterritt and D. Bustard, "Towards an Autonomic Computing Environment," In Proc. of 14th IEEE Int'l Workshop on Database and Expert Systems Applications, September 2003.

[3] R. M. Alexander, "Energy for Animal Life," Oxford University Press, May 1999.

[4] P. Champrasert and J. Suzuki, "SymbioticSphere: A Biologically-inspired Network Architecture for Autonomic Grid Systems," Proc. of IASTED CIIT, October 2005.

[5] J. Suzuki and T. Suda, "A Middleware Platform for a Biologically-inspired Network Architecture Supporting Autonomous and Adaptive Applications" IEEE J. on Selected Areas in Comm. Feb. 2005.

[6] P. Gunaratne, K. Christensen, and B. Nordman, "Managing Energy Consumption Costs in Desktop PCs and LAN Switches with Proxying, Split TCP connections, and Scaling of Link Speed," Int'l J. of Network Management, Vol. 15, No. 5, 2005.

[7] Advanced Micro Devices, Inc., Magic Packet Technology, Technical White Paper 20213, November 1995.

[8] C. Lefurgy, K. Rajamani, F. Rawson, W. Felter, M. Kistler, and T. W. Keller. "Energy management for commercial servers," IEEE Computer, 36(12):39-48, December 2003.

[9] P. Champrasert and J. Suzuki, "SymbioticSphere: A Biologically-Inspired Autonomic Architecture for Self-Adaptive and Self-Healing Server Farms," Proc. of IEEE Int'l Workshop on Autonomic Communications and Computing, June 2006.

[10] P. Champrasert and J. Suzuki, "A Biologically-Inspired Autonomic Architecture for Self-Healing Data Centers," Proc. of IEEE International Conference on Computer Software and Applications Conference, September 2006.

[11] N. Wakamiya and M. Murata, "Toward Overlay Network Symbiosis," In Proc. of P2P 2005, September 2005.

[12] Junjiro Konishi, Naoki Wakamiya and Masayuki Murata, "Proposal and evaluation of a cooperative mechanism for pure P2P file sharing networks," Proc. of International Workshop on Biologically Inspired Approaches to Advanced In-formation Technology, January 2006.

[13] S. Cheng, D. Garlan, B. Schmerl, P. Steenkiste and N. Hu, "Software Architecture-based Adaptation for Grid Computing," Proc. of IEEE HPDC, July 2002.

[14] C. Adam and R. Stadler, "Adaptable Server Clusters with QoS Objectives," Proc. of IFIP/IEEE IM, May, 2005. 